\title{
Author Correction: Artificial water channels enable fast and selective water permeation through water-wire networks
}

Woochul Song, Himanshu Joshi, Ratul Chowdhury (D), Joseph S. Najem, Yue-xiao Shen, Chao Lang, Codey B. Henderson, Yu-Ming Tu, Megan Farell, Megan E. Pitz, Costas D. Maranas, Paul S. Cremer, Robert J. Hickey, Stephen A. Sarles, Jun-li Hou, Aleksei Aksimentiev (D) and Manish Kumar (iD)

Correction to: Nature Nanotechnology https://doi.org/10.1038/s41565-019-0586-8, published online 16 December 2019.

In the version of this Article originally published, the authors mistakenly failed to include the following key reference: Chen, L. et al. Chiral selective transmembrane transport of amino acids through artificial channels. J. Am. Chem. Soc. 135, 2152-2155 (2013). This has now been added as ref. 12 and subsequent references have been renumbered. The new reference has been cited in the following sentences: (1) "Achieving both AQP-like single channel water permeability..."; (2) "It has been reported that the permeability of..."; (3) “These results are similar to our recent study that demonstrated..."; and (4) "Therefore, the unimolecular AWC PAP[5]..., as well as in Fig. 2c caption "Comparison of the calculated single channel permeability..." These changes have been made in the online versions of the Article.

Published online: 24 January 2020

https://doi.org/10.1038/s41565-020-0640-6

(๑) The Author(s), under exclusive licence to Springer Nature Limited 2020 